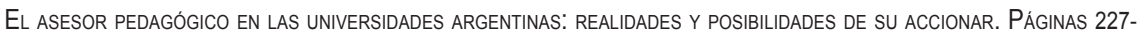
246 en Revista de la Escuela de Ciencias de la EduCAción, año 10, Número 9, enero a diciembre de 2014. ISSN 1851-6297. ISSN EN LINEA 2362-3349.

\title{
EL ASESOR PEDAGÓGICO EN LAS UNIVERSIDADES ARGENTINAS: REALIDADES Y POSIBILIDADES DE SU ACCIONAR
}

\author{
Por Elisa Lucarelli (Universidad de Buenos Aires)*, Argentina. \\ elisalucarelli@arnet.com.ar \\ Claudia Finkelstein (Universidad de Buenos Aires)**, Argentina. \\ claudiafinkelstein@yahoo.com.ar \\ Viviana Solberg (Universidad de Buenos Aires) ${ }^{* * *}$, Argentina. \\ vivisolberg@yahoo.com.ar
}

Recibido: 04/07/2014 Aceptado: 07/11/2014

\section{Resumen}

Este artículo presenta los avances producidos en la investigación desarrollada por el equipo de la Universidad de Buenos Aires - integrantes del Programa de Investigación Estudios sobre el aula universitaria de la Facultad de Filosofía y Letras de esa Universidad - centrado en análisis de las unidades de asesorías pedagógicas universitarias.

Ubicado en el marco del Proyecto Conjunto de Investigación Mercosur Argentina (SPU)- Brasil, (CAPES) PPCP 003/11, Estrategias institucionales para el mejoramiento de la calidad de la Educación Superior y el desarrollo profesional docente, con sedes en la UNISINOS por Brasil y en la UBA por Argentina, este equipo desarrolla las acciones de indagación sistemática.

\footnotetext{
* Doctora en Educación, Licenciada y Profesora en Ciencias de la Educación de la Universidad de Buenos Aires. Profesora de Posgrado e investigadora en la UNTREF. Es Investigadora Categoría I y Directora del Programa Estudios sobre el aula universitaria (IICE,FFyL, UBA).

** Licenciada y Profesora en Ciencias de la Educación de la Universidad de Buenos Aires. Especialista en Formación de Formadores y Magister en Formación de Formadores. FFyL- UBA. Doctoranda de la Universidad de Buenos Aires.

*** Licenciada y Profesora en Ciencias de la Educación (UBA), Especialista en Gestión y Docencia para el Nivel Superior (Universidad Nacional de Tres de Febrero), Maestría en Políticas y Administración de la Educación (en curso, Universidad Nacional de Tres de Febrero.
} 
Revista de la Escuela de Ciencias de la Educación, año 10, número 9, enero a diciembre de 2014. Páginas 227-246. ISSN 1851-6297. ISSN EN LINEA 2362-3349. EL ASESOR PEDAGÓGICO EN LAS UNIVERSIDADES ARGENTINAS: REALIDADES Y POSIBILIDAdES DE SU ACCIONAR. Elisa LuCARELLI - Claudia FinKELstein - Viviana Solberg.

Aquí se presentan los avances en cuanto al análisis de las experiencias de asesorías pedagógicas universitarias en la Etapa I de esta investigación. En ella se trabajó con información de material documental, analizando 20 ponencias presentadas por Universidades Nacionales argentinas en el II Encuentro Nacional de Prácticas de Asesorías Pedagógicas Universitarias realizado en la Ciudad de Buenos Aires en el año 2010.

Los aportes se orientan a la comprensión de la problemática central alrededor de la cual estas unidades de pedagogía universitaria nuclean sus actividades.

\title{
Palabras Clave
}

Asesor pedagógico universitario - Educación superior - Estrategias de intervención - Problemáticas centrales.

\begin{abstract}
This paper presents the progress made in the research conducted by the team from the University of Buenos Aires - members of the Research Program "Studies in the university classroom" in the Faculty of Arts at that University - focused on the analysis of university pedagogical advice units.

Located in the framework of the Joint Research Project Mercosur Argentina (SPU) - Brazil, (CAPES) PPCP 003/11, "Institutional strategies for improving the quality of higher education and the professional development of teachers", based in UNISINOS (Brazil) and UBA (Argentina), this team develops systematic research actions.

This paper presents advances in analyzing the experiences of university pedagogical advisory in Phase I of this research. We worked with documentary material information, analyzing 20 papers presented by Argentinean National Universities in the II National Pedagogical Practices of University Advising held in Buenos Aires in 2010. The contributions target understanding the central issue around which these university teaching units nucleate their activities.
\end{abstract}

\section{Key Words}

Pedagogical adviser - Higher education - University teaching - Institutional strategies - Professional development.

\section{A modo de encuadre}

Este artículo presenta los avances producidos en la investigación desarrollada por el equipo de la Universidad de Buenos Aires - integrantes del Programa de Investigación Estudios sobre el aula universitaria de la Facultad de Filosofía y Letras de esa Universidad - centrado en análisis de las unidades de asesorías pedagógicas universitarias.

Ellas han desarrollado, en las últimas décadas, un significativo papel como uno de los recursos que las universidades de Argentina y de otros países latinoamericanos están llevando a cabo en la búsqueda de acciones potentes y genuinas para el mejoramiento de la calidad de la educación superior. 
Revista de la Escuela de Ciencias de la Educación, año 10, número 9, enero a diciembre de 2014. Páginas 227-246. ISSN 1851-6297. ISSN EN LINEA 2362-3349. EL ASESOR PEDAGÓGICO EN LAS UNIVERSIDADES ARGENTINAS: REALIDADES Y posibilidades de su acCiOnar. Elisa LuCARELli - Claudia Finkelstein - Viviana Solberg.

Estas líneas de acción, si bien no contradicen los propósitos de los programas de evaluación y acreditación de carreras e instituciones que ocupan la agenda a nivel mundial, intentan desarrollar y apoyar líneas centradas en la idiosincrasia del aula y del contexto institucional, de manera de contribuir a generar y desarrollar proyectos situados de innovación y mejoramiento de la formación de los estudiantes.

El proyecto que desde el año 2011 desarrollan equipos de Argentina y Brasil dedicados a la Pedagogía y Didáctica universitarias, se orienta por estos principios, concretando la formalización de intercambios académicos sostenidos por ellos desde hace más de veinte años. Se trata del Proyecto Conjunto de Investigación Mercosur Argentina (SPU)- Brasil, (CAPES) PPCP 003/11, Estrategias institucionales para el mejoramiento de la calidad de la Educación Superior y el desarrollo profesional docente, con sedes en la UNISINOS por Brasil y en la UBA por Argentina, el cual, desarrolla las acciones de indagación sistemática y formación de posgrado previstas en ese Proyecto teniendo en consideración las orientaciones arriba mencionadas.

A su vez, en cada país el proyecto articula en torno al tema de las estrategias para el mejoramiento de la calidad en el nivel, las actividades de distintas instituciones universitarias. En el caso de Argentina, implica el trabajo conjunto, coordinado por la UBA, de un grupo integrado por universidades nacionales de este país, la Universidad Nacional de Tucumán, la Universidad Nacional del Sur y la Universidad Nacional de Entre Ríos, a las que se suma la Universidad de la República, de Uruguay. Los cinco equipos institucionales de universidades argentinas y uruguaya involucrados en el Proyecto están encarando de manera idiosincrásica esta indagación.

En el contexto universitario actual, signado por tensiones y diversas representaciones institucionales, la asesoría pedagógica universitaria se hace presente como uno de los recursos posibles a los que la institución puede acudir para emprender procesos de transformación en el campo de la enseñanza.

En el caso de Argentina y Uruguay, que enfrentan desde hace más tiempo la compleja ecuación entre la ampliación del acceso y la calidad de la educación superior, se implementaron algunos dispositivos que fueron dándole a la Pedagogía y la Didáctica universitarias, un cierto espacio de legitimidad, desde la perspectiva que auxilia a la comprensión de los desafíos y propone caminos alternativos.

En estas universidades los espacios fueron transformados en lugares, (Da Cunha, 2010) en la medida en que se crearon, institucionalmente, las asesorías pedagógicas en las diferentes carreras y facultades, con miras a producir conocimiento sobre los procesos de enseñar propios de las culturas profesionales disciplinares y en las condiciones objetivas de cada área del saber (Da Cunha y Lucarelli, 2011). 
Revista de la Escuela de Ciencias de la Educación, año 10, número 9, enero a diciembre de 2014. Páginas 227-246. ISSN 1851-6297. ISSN EN LINEA 2362-3349. EL ASESOR PEDAGÓGICO EN LAS UNIVERSIDADES ARGENTINAS: REALIDADES Y posibilidades de su aCcIONAR. Elisa LuCARELli - Claudia Finkelstein - Viviana Solberg.

Estas asesorías contaron con el aporte de profesionales del área de educación que asumían tareas de investigación y apoyo pedagógico a los docentes y a la gestión académica.

El equipo UBA se propuso caracterizar a las asesorías pedagógicas universitarias (APU) en cuanto a su descripción y problemáticas centrales, desarrollándolo en dos etapas:

En la Etapa I se trabajó con información de material documental. En este sentido, se abordó el análisis de las ponencias presentadas en el II Encuentro Nacional de Prácticas de Asesorías Pedagógicas Universitarias. Se realizó el análisis transversal de 20 ponencias presentadas por 24 Unidades Pedagógicas de las siguientes Universidades Nacionales argentinas: Universidad Nacional del Litoral, Universidad Nacional de Rosario, Universidad de Buenos Aires, Universidad Nacional de Entre Ríos, Universidad Nacional del Sur, Universidad Nacional de Río Cuarto, Universidad Nacional de la Patagonia San Juan Bosco, Universidad Nacional del Litoral, Universidad Nacional de San Juan, Universidad Nacional de La Plata, RUNCOB - Red de Universidades del Conurbano Bonaerense (Universidad Nacional de La Matanza, Universidad Nacional de General Sarmiento, Universidad Nacional de Lanús y Universidad Nacional de Tres de Febrero) y la Universidad Nacional de Tucumán.

En la Etapa II (en proceso de elaboración final) se trabajó con información obtenida a través de entrevistas en profundidad a Asesores Pedagógicos Universitarios de una Unidad Académica de la UBA y de las Universidades Nacionales de Luján, Río Cuarto, Rosario y General Sarmiento, con el propósito de recabar información y analizar otros aspectos relacionados con la visión que los propios sujetos tienen sobre el desempeño de su rol.

En este artículo se presentan algunas conclusiones de la Etapa I, referidas a las actividades centrales que desarrollan los Asesores Pedagógicos Universitarios.

En primer término se identificaron los siguientes tipos de actividades desempeñados por el asesor pedagógico universitario: formación, apoyo a proyectos institucionales, investigación, extensión y asesoramiento.

En segundo término, se relevaron las frecuencias con que el asesor desarrolla estas tareas. Esto se expresa en el siguiente cuadro. 
Revista de la Escuela de Ciencias de la Educación, año 10, número 9, enero a diciembre de 2014. Páginas 227-246. ISSN 1851-6297. ISSN EN LINEA 2362-3349. EL ASESOR PEDAGÓGICO EN LAS UNIVERSIDADES ARGENTINAS: REALIDADES Y posibilidades de SU ACCIONAR. Elisa LuCARElli - Claudia FinKelstein - Viviana Solberg.

\section{Cuadro № 1: Problemática central}

\begin{tabular}{|c|c|}
\hline Tipo de actividades & Frecuencia \\
\hline Apoyo a proyectos institucionales & 33 \\
\hline Formación & 25 \\
\hline Asesoramiento & 17 \\
\hline Investigación & 13 \\
\hline Extensión & 3 \\
\hline Total & 91 \\
\hline
\end{tabular}

Se ha observado que el rol que más frecuentemente lleva a cabo el APU es el apoyo a proyectos institucionales (con una frecuencia de 33 menciones), donde encontramos que desarrollan actividades para favorecer la retención de los estudiantes, para favorecer el ingreso, de orientación vocacional y otros de carácter general. El cuadro que sigue permite advertir los énfasis con que se llevan a cabo este tipo de tareas.

Cuadro $N^{\circ}$ 2: Apoyo a proyectos institucionales

\begin{tabular}{|c|c|}
\hline Modalidad & Frecuencia \\
\hline Para favorecer el ingreso & 8 \\
\hline Para favorecer la retención & 17 \\
\hline Orientación vocacional & 7 \\
\hline Otras modalidades & 1 \\
\hline Total & 33 \\
\hline
\end{tabular}

En segundo, lugar lleva adelante la formación del docente de este nivel $y$ de estudiantes (F: 25), desarrollando diverso tipo de dispositivos en función de variados destinatarios. 
Revista de la Escuela de Ciencias de la Educación, año 10, número 9, enero a diciembre de 2014. PÁginas 227-246. ISSN 1851-6297. ISSN EN LINEA 2362-3349. EL ASESOR PEDAGÓGICO EN LAS UNIVERSIDADES ARGENTINAS: REALIDADES Y POSIBILIDAdES DE SU ACCIONAR. Elisa LuCARELLI - Claudia FinKELstein - Viviana Solberg.

\section{Cuadro N³: Formación}

\begin{tabular}{|c|c|c|c|c|}
\hline Tipo & Destinatario & Modalidad & Frecuencia & Total \\
\hline \multirow{8}{*}{$\begin{array}{l}\text { En el rol } \\
\text { docente }\end{array}$} & \multirow{4}{*}{$\begin{array}{l}\text { Para docentes } \\
\text { propiamente } \\
\text { dichos }\end{array}$} & Espacios de reflexión & 3 & \multirow[t]{4}{*}{18} \\
\hline & & Cursos & 11 & \\
\hline & & Posgrados & 2 & \\
\hline & & Otras modalidades & 2 & \\
\hline & $\begin{array}{l}\text { Para docentes } \\
\text { noveles } \\
\text { (alumnos o } \\
\text { graduados) }\end{array}$ & & & 3 \\
\hline & \multirow[t]{3}{*}{ Para tutores } & Espacios de reflexión & 1 & \multirow[t]{3}{*}{3} \\
\hline & & Cursos & 1 & \\
\hline & & Otras modalidades & 1 & \\
\hline \multicolumn{3}{|c|}{ De estudiantes de grado (en el marco de una carrera) } & 1 & 1 \\
\hline \multicolumn{3}{|c|}{ Total } & & 25 \\
\hline
\end{tabular}

En tercer término, el asesoramiento ( $F: 17)$, fundamentalmente a cátedras y en cuestiones de acreditación o autoevaluación, tal como se observa en el cuadro que sigue:

Cuadro $N^{\circ} 4$ : Asesoramiento

\begin{tabular}{|l|l|c|c|}
\hline \multicolumn{1}{|c|}{ Modalidad } & \multicolumn{1}{|c|}{ Temáticas } & Frecuencia & Total \\
\hline A cátedras & & 5 & 5 \\
\hline \multirow{2}{*}{ A la gestión } & $\begin{array}{l}\text { En cuestiones de } \\
\text { acreditación o autoevaluación }\end{array}$ & 5 & \\
\cline { 2 - 4 } & Otros & 7 & 12 \\
\hline Total & & 17 & 17 \\
\hline
\end{tabular}


Revista de la Escuela de Ciencias de la Educación, año 10, número 9, enero a diciembre de 2014. Páginas 227-246. ISSN 1851-6297. ISSN EN LINEA 2362-3349. EL ASESOR PEDAGÓGICO EN LAS UNIVERSIDADES ARGENTINAS: REALIDADES Y posibilidades de su acCiOnar. Elisa LuCARELli - Claudia Finkelstein - Viviana Solberg.

Otra de las tareas que realiza en menor medida el asesor es la investigación. Respecto a ésta última, (F: 13) se observan énfasis vinculados a respuestas a la demanda institucional, como modalidad estratégica, en cuanto a la conformación de los equipos y utilizando metodología cualitativa y como objeto de estudio el campo de la Pedagogía y Didáctica Universitarias.

En último lugar aparecen tareas de extensión (F:3) con una frecuencia mucho menor.

A continuación se analiza cada una de las actividades que cotidianamente llevan a cabo los Asesores Pedagógicos Universitarios.

\section{Acerca del Desarrollo de proyectos institucionales}

Una de las actividades que detectamos con mayor fuerza dentro del desempeño de las unidades de Asesoría Pedagógica Universitaria analizadas es el desarrollo de distintos proyectos institucionales. Como ya se ha señalado, se realizan 33 menciones a este tipo de actividad. La gran mayoría de estos proyectos tienen como eje central el acompañamiento a los estudiantes en distintos aspectos o momentos de su trayecto formativo: encontramos, por un lado, proyectos que se proponen favorecer el ingreso de los estudiantes a la vida universitaria; por otro lado, proyectos que pretenden facilitar, a través de distintos dispositivos, la retención de los alumnos una vez que pasaron las primeras etapas de su trayecto formativo en la universidad; y por último, proyectos que hacen foco en la orientación vocacional. Muchos de esos proyectos hacen eje en más de uno de esos objetivos.

Como modo de paliar el fenómeno de la deserción y del fracaso temprano muchas instituciones deciden actualmente destinar esfuerzos tendientes a acompañar a los estudiantes en su trayecto formativo especialmente en los primeros años.

Entre las líneas de acción y dispositivos institucionales implementados para intervenir en ese sentido, es posible mencionar, a modo de ejemplo, los cursos preparatorios y/o de ingreso, las acciones de bienvenida, las charlas informativas para alumnos ingresantes, la producción de material informativo, los talleres con temáticas diversas asociadas al ingreso, etc. Entre estas acciones, merecen una mención especial los sistemas de tutoría que, en los últimos años, comenzaron a implementarse de manera creciente en las universidades del país, tanto por iniciativa de las unidades académicas como promovidas, en ciertos casos, por organismos educativos centrales.

Los sistemas tutoriales llevan adelante acciones que, a través de distintos dispositivos, hacen foco en el sujeto, ofreciéndole acompañamiento, sostén y guía. El sujeto es concebido como un individuo que lleva adelante un proyecto personal de formación que supone el pasaje de la adolescencia a la adultez, en términos de la búsqueda de un lugar en el mundo de los adultos y del trabajo. Según Canessa (2002), en Larramendy y otras (2012), transitar este pasaje 
Revista de la Escuela de Ciencias de la Educación, año 10, número 9, enero a diciembre de 2014. Páginas 227-246. ISSN 1851-6297. ISSN EN LINEA 2362-3349. EL ASESOR PEDAGÓGICO EN LAS UNIVERSIDADES ARGENTINAS: REALIDADES Y posibilidades de su aCcIONAR. Elisa LuCARELli - Claudia Finkelstein - Viviana Solberg.

conlleva una cuota de sufrimiento psíquico vinculado al momento de duelo por la infancia perdida, la sacudida de las identificaciones que conduce a la búsqueda de un nuevo lugar, y la asunción de nuevas responsabilidades.

La incorporación al mundo universitario supone inevitablemente sentimientos de ansiedad e incertidumbre, ligados al contacto con lo desconocido: en ese nuevo contexto el alumno deberá aprender el "oficio de ser estudiante".

De este modo Larramendy y otras (2012) señalan que las intervenciones diseñadas en los sistemas tutoriales, en sus variadas modalidades (de pares, llevadas a cabo por docentes, grupales, individuales, obligatorias o a demanda), se proponen acompañar y facilitar mejores condiciones para que los sujetos 'aprenden a ser estudiantes' y a 'estar en la universidad', en el trayecto que se sabe, puede ser el más crítico: el inicio de la vida universitaria.

En el marco de estas acciones institucionales, los equipos APU se constituyen como actores fundamentales para su desarrollo.

Las diferentes ponencias analizadas en este eje ponen de manifiesto que los equipos de Asesoría Pedagógica se abocan cada vez más al abordaje de estas dificultades y a la implementación de propuestas de intervención que contribuyan a paliarlas, procurando favorecer la retención y el avance regular en los estudios: indagación de los factores que inciden en el bajo rendimiento, vinculación con la escuela media, servicio de orientación vocacional-ocupacional, revisión del régimen de cursada y evaluación, dictado de cursos de formación disciplinaria complementaria, espacios para la socialización en la institución universitaria, y especialmente la implementación de sistemas de tutorías.

Analizando la situación expresada por las Unidades Pedagógicas a través de las ponencias se puede apreciar que la mayoría de estos proyectos tienen al estudiante universitario como destinatario principal, y en particular a sus dificultades para ingresar o avanzar en el trayecto formativo. Si bien esto parece evidente, ya que es el destinatario final y natural de toda acción universitaria que favorezca la formación, tradicionalmente fue pensado como el culpable de su fracaso. Proyectos que intentan comprender las dificultades por las que atraviesa el estudiante y proponen medidas para paliarlas suponen una nueva toma de posición por parte de la institución universitaria, que de alguna manera está señalando que se hace cargo de dichas dificultades.

Es el ejemplo de la unidad de asesoría de la Facultad de Ciencias Agrarias de la Universidad Nacional de Rosario que organizó un equipo de tutores pares que basó su accionar en la detección de problemáticas, acompañamiento y orientación del alumno universitario. Si bien fue concebido con el objetivo de favorecer el ingreso y la retención, por motivos presupuestarios, la implementación de Tutorías sólo pudo realizarse en el Ingreso y Primer año. El testimonio que sigue advierte que:

Se tuvo en cuenta tanto la adaptación social al medio universitario como lo relacionado a la construcción de estrategias de estudio au- 
Revista de la Escuela de Ciencias de la Educación, año 10, número 9, enero a diciembre de 2014. Páginas 227-246. ISSN 1851-6297. ISSN EN LINEA 2362-3349. EL ASESOR PEDAGÓGICO EN LAS UNIVERSIDADES ARGENTINAS: REALIDADES Y posibilidades de su acCiOnar. Elisa LUCARELLI - Claudia Finkelstein - Viviana Solberg.

tónomo y comprensivo. Para ello se realizaron desde la Asesoría Pedagógica acciones puntuales, fundamentalmente en el Curso de Ingreso a través de encuestas, sondeos y análisis de producciones. (Facultad de Ciencias Agrarias de la Universidad Nacional de Rosario)

Cabe señalar que en muchos casos un mismo equipo APU desarrolla acciones orientadas a más de uno de los propósitos mencionados (ingreso, retención, orientación vocacional) utilizando más de un dispositivo.

Como otro tipo de acción, una APU (el Servicio Pedagógico Universitario de la Facultad de Ciencias Bioquímicas y Farmacéuticas de la UNR) elaboró una estrategia de trabajo docente interdisciplinario como alternativa para superar dificultades vinculadas al examen final de estudiantes de una asignatura: se planteó una intervención cuyo propósito que direcciona la acción intenta fortalecer estrategias docentes y el trabajo con los alumnos para superar las dificultades al momento de rendir nuevamente la asignatura. Involucrados en la experiencia estuvieron profesores disciplinarios y el equipo APU.

Otra acción está centrada en un Programa de Orientación de la Facultad de Filosofía y Letras de la Universidad de Buenos Aires que ofrece información, asesoramiento y asistencia administrativa, pedagógica y vocacional ocupacional tanto a los aspirantes, los estudiantes de grado, como los graduados de las carreras que se cursan en esa Facultad. Además de la línea de trabajo centrada en la Información y Asistencia (que se basa en el ciclo de charlas informativas para los alumnos ingresantes, la atención de consultas, el asesoramiento y asistencia a docentes y/o autoridades de los Departamentos de las distintas carreras, el Espacio de Orientación Pedagógica para alumnos del grado -donde se trabajan las dificultades que pueden aparecer en el proceso de aprendizaje- y el Proyecto "Alumnos avanzados tutores de alumnos ingresantes"), el Programa lleva adelante acciones de vinculación con escuelas medias a través de visitas de las mismas a la Facultad desarrolla dos grandes líneas de acción enmarcadas en la línea de trabajo relacionada con la Difusión.

Otra APU, en función de demandas de rectorado, desarrolló una investigación para dar cuenta de los factores intervinientes en la deserción, de manera de contar con información para la toma de decisiones.

Como se puede observar, la mayoría de los equipos hace foco en la problemática de los estudiantes a través de la puesta en marcha de distintas dispositivos, entre las que predominan los sistemas de tutorías generalmente articulados con otras acciones complementarias, tales como talleres o instancias de orientación. También se aprecian intenciones de comprender más a fondo las cuestiones relacionadas con el ingreso y la permanencia (o, por el contrario, el abandono), a través de acciones tendientes a recabar y analizar la información correspondiente. 
Revista de la Escuela de Ciencias de la Educación, año 10, número 9, enero a diciembre de 2014. Páginas 227-246. ISSN 1851-6297. ISSN EN LINEA 2362-3349. EL ASESOR PEDAGÓGICO EN LAS UNIVERSIDADES ARGENTINAS: REALIDADES Y posibilidades de su aCcIONAR. Elisa LuCARELli - Claudia Finkelstein - Viviana Solberg.

Por último, señalemos que la bibliografía especializada llama la atención acerca de la necesidad de vincular las acciones centradas en el estudiante y sus dificultades, con aquellas centradas en la enseñanza y su perfeccionamiento. Larramendy y otras (2012) sostienen que resulta necesario advertir y reflexionar sobre los límites de los tipos de intervención centrados en el sujeto para revertir los fenómenos de abandono y desgranamiento y contribuir a hacer efectivas las intenciones democratizadoras de los procesos de apertura de las instituciones universitarias, interrogándose si es posible favorecer los procesos de formación de los estudiantes mediante acciones de apoyo centradas en el sujeto de aprendizaje sin cuestionar, revisar o introducir modificaciones en las propuestas de enseñanza universitaria y las condiciones mediante las que intentan promover los aprendizajes de los alumnos.

Presumiblemente concientes de esta necesidad, los equipos APU analizados combinan muchas veces ambos tipos de acciones. De hecho varios equipos desarrollan paralelamente tareas de formación docente, así como de acompañamiento estudiantil, en cualquiera de sus versiones.

Uno de los equipos lo señala de la siguiente manera:

Desde nuestra experiencia, las acciones tutoriales disociadas de las prácticas docentes en las asignaturas, presentan limitaciones. Por ello insistimos en que es imprescindible partir del intercambio de información y perspectivas entre la Tutoría y los Equipos Docentes (Facultad de Ciencias Agrarias de la Universidad Nacional de Rosario).

\section{Acerca de las acciones de formación}

Del análisis de las 24 Unidades Pedagógicas presentado a través de las ponencias surge que uno de los roles que desempeña el asesor pedagógico universitario frecuentemente es la formación del docente de este nivel. Esta tarea se conforma como un verdadero desafío para el APU. La formación de docentes universitarios es hoy en día, sin lugar a dudas, eje de preocupación en todas partes del mundo. El profesor universitario es un profesional, especialista de alto nivel que se dedica a la enseñanza y es miembro de una comunidad académica. Participa en programas formativos cuyo objetivo es fomentar cambios en sus prácticas docentes.

Es indudable que la preocupación por la formación de los docentes en el nivel superior es relativamente reciente. La experticia profesional, la especialidad en un campo de conocimientos, la experiencia en la profesión y en la docencia, y en especial los antecedentes en investigación vehiculizados a través de las publicaciones científicas, fueron tradicionalmente los requerimientos necesarios para acceder a la docencia universitaria. 
Revista de la Escuela de Ciencias de la Educación, año 10, número 9, enero a diciembre de 2014. Páginas 227-246. ISSN 1851-6297. ISSN EN LINEA 2362-3349. EL ASESOR PEDAGÓGICO EN LAS UNIVERSIDADES ARGENTINAS: REALIDADES Y posibilidades de su acCiOnar. Elisa LuCARELli - Claudia Finkelstein - Viviana Solberg.

En los últimos años, la formación pedagógica de los docentes universitarios ha tenido un creciente interés a partir del reconocimiento de su impacto en la calidad de los aprendizajes de los estudiantes. En este sentido, el fracaso del estudiantado ha dejado de ser responsabilidad única del alumno, incorporando otras variables, entre ellas, el sistema, la institución y el docente.

En términos generales la literatura referida al tema sugiere que, a diferencia de otros niveles del sistema educativo, la formación del docente universitario se centra en un profesor que ya se encuentra en el ejercicio profesional de la enseñanza en diferentes momentos de su trayecto como docente. Sin embargo, el docente novel, que accede a la docencia en el nivel, ha pasado por instancias de formación al interior de la cátedra de origen, en un proceso que Ickowicz (2002) denomina modelo artesanal. Se caracteriza a grandes rasgos por elección mutua entre el maestro y el discípulo, las prácticas de enseñanza se adecuan a las dificultades que se van presentando, se basa en la experiencia y la enseñanza y aprendizaje se desarrollan en el propio ámbito de producción. Es decir, que las prácticas de enseñanza, se van configurando en un modelo que no difiere grandemente del status quo ya establecido y cuyas particularidades derivan de estilos personales. Son secretos del oficio que el aprendiz va adquiriendo al realizar determinadas tareas y estar cerca del maestro, que es quién garantiza el acceso al conocimiento y la continuidad de la tarea. El entramado entre trabajo (como docente) y formación va generando procesos fuertes de socialización. Esto implica que sus modos de actuación responden a lógicas de continuidad con modelos docentes preestablecidos, que nos son sujetos a reflexión.

La formación es uno de los elementos que integran el desarrollo profesional del docente universitario; la carrera docente, el status profesional, el sistema retributivo, el clima laboral, el contexto laboral, etc. son otros factores que lo componen.

Sánchez Núñez (2001) define el desarrollo profesional del docente universitario como:

- un proceso continuo que se desarrolla durante toda la vida profesional que no puede entenderse como actividades aisladas ineficaces.

- basado en la mejora profesional, centrándose en las necesidades reales que tienen los docentes.

- llevado a cabo mediante la participación tanto en el diseño de la innovación como en la toma de decisiones para el trabajo profesional.

Es un proceso de construcción profesional en donde, a través de las reflexiones sobre la problemática de la enseñanza, el profesor de este nivel de enseñanza va desarrollando destrezas cognitivas y metacognitivas que le permiten realizar modificaciones en sus prácticas y la valoración de su trabajo profesional. 
Revista de la Escuela de Ciencias de la Educación, año 10, número 9, enero a diciembre de 2014. Páginas 227-246. ISSN 1851-6297. ISSN EN LINEA 2362-3349. EL ASESOR PEDAGÓGICO EN LAS UNIVERSIDADES ARGENTINAS: REALIDADES Y posibilidades de su aCcIONAR. Elisa LuCARELli - Claudia Finkelstein - Viviana Solberg.

En el análisis de las ponencias presentadas en relación con la formación, en las 25 actividades presentadas por las APU, hemos discriminado dos grandes categorías: una referida a la formación en el rol docente, y otra vinculada con la formación de estudiantes de grado.

Vamos a referirnos en primer lugar a la formación en el rol docente. En esta categoría discriminamos tres tipos de destinatarios: para docentes propiamente dichos, para docentes nóveles (alumnos o graduados) y para tutores. En cada uno se han observado diferentes dispositivos llevados a cabo para su formación.

En primer lugar, se desarrollan espacios de reflexión destinados a la formación de docentes propiamente dichos. Se caracterizan por ser encuentros no estructurados, centrados alrededor de una problemática que analiza y reflexiona sobre lo acontecido en el aula. Los espacios de reflexión, como dispositivos de formación del profesor universitario se constituyen en privilegiados para la explicitación y el trabajo de reconfiguración de las teorías de las que son portadores los docentes. Basados en el paradigma crítico -reflexivo, remarcan el protagonismo del docente al poner en análisis sus propias prácticas de enseñanza.

En esta línea, otras propuestas formativas adoptan la modalidad de taller. En el caso del presente análisis, estos espacios, en sus diferentes modalidades permiten el trabajo con problemáticas que los docentes de cada institución en sus prácticas cotidianas, afrontan. Es un ámbito de reflexión y de acción que supera la disociación entre la teoría y la práctica, en donde la primera refiere a una práctica concreta que se presenta como problema, ilumina la práctica interpretando lo realizado u reorientando la acción, son dos polos en permanente relación. Su objetivo es la producción de nuevos conocimientos. Así lo expresan los protagonistas: "(...) un espacio de reflexión pedagógica y de análisis en torno a las prácticas universitarias de enseñanza y evaluación" (UNER. Facultad de Ingeniería. Área de Asesoría Pedagógica y de Orientación Vocacional).

Otro tipo de experiencias formativas, destinadas a la formación de docentes propiamente dichos lo constituyen los cursos. Estos son espacios sistemáticos y estructurados que ofrecen 11 de las diferentes unidades pedagógicas. Algunos de ellos cumplen una función compensatoria instrumental ya que se orientan a aspectos más ligados al entrenamiento que a la formación.

Por otro lado, la formación de docentes propiamente dichos se aborda a través de programas sistemáticos de Posgrado. Si bien la frecuencia con que se presentan estos programas es baja (dos Unidades Académicas lo desarrollan), los mismos forman parte del crecimiento de los Posgrados en nuestro país, y en especial los dedicados a la formación en docencia de los cuadros de docentes universitarios.

Las otras acciones de formación tienen como destinatarios a los docentes noveles (alumnos o graduados). Se trata de modalidades que se proponen 
Revista de la Escuela de Ciencias de la Educación, año 10, número 9, enero a diciembre de 2014. Páginas 227-246. ISSN 1851-6297. ISSN EN LINEA 2362-3349. EL ASESOR PEDAGÓGICO EN LAS UNIVERSIDADES ARGENTINAS: REALIDADES Y posibilidades de su acCiOnar. Elisa LuCARELli - Claudia Finkelstein - Viviana Solberg.

el abordaje de cuestiones pedagógicas para docentes sin experiencia, que realizan sus primeras tareas de formación en el ámbito universitario -más allá de las realizadas a través del modelo artesanal, a través de: pasantías, espacios de reflexión sobre sus prácticas y seminarios y cursos sobre Pedagogía universitaria.

El tercer tipo de destinatario de acciones de formación en el rol docente, se refiere a la formación de tutores, que es desarrollada por tres de las instituciones estudiadas. Se trata de espacios de reflexión y cursos.

Por último, destacamos un nuevo destinatario en el proceso formativo: el estudiante, encontrándose un caso de APU que manifiesta desarrollar espacios de formación de estudiantes de grado (en el marco de una carrera).

A partir del análisis realizado se observa que, en términos generales, las acciones de formación se encuentran aisladas y poco articuladas. Muchas de ellas se orientan a resolver problemas específicos detectados de diversas maneras, por tanto, su carácter puede ser asistencial. Este tipo de estrategia tiende a solucionar situaciones inmediatas, sin que impliquen necesariamente un plan de acción articulado institucionalmente. De este modo, la oferta que más se presenta la constituyen espacios de reflexión, cursos y otras modalidades - destinadas tanto a docentes que ya se desempeñan como tales, como a los que recién inician su trayectoria- que en algunos casos se repiten a lo largo del tiempo. Sin embargo, los mismos podrían constituirse en la primera etapa que podría ser complementada con una capacitación sistemática ofrecida por programas formales.

\section{Acerca de las tareas de asesoramiento}

En esta dimensión de análisis de la investigación se alude al asesoramiento en un doble sentido: como la actividad que refiere, da nombre, y por tanto marca de manera significativa al rol del asesor pedagógico en la institución, y como núcleo de tareas específicas relativas a una de las áreas de desempeño de este actor.

En esta búsqueda de comprensión del rol, la asesoría pedagógica es reconocida como una profesión de ayuda en un medio donde las prácticas de intervención se orientan a lograr cambios que afecten a la institución universitaria como un todo y al aula en particular. De allí que se entienda al asesoramiento enfocando los procesos interactivos de colaboración con las instituciones para prevenir posibles problemas, participar y ayudar en la solución de los que puedan existir y cooperar en la consecución de una mayor mejora educativa. (Murillo Estepa, 1997: 44). Considerado con un recurso estratégico para el cambio, su accionar implica esclarecimiento de la situación y búsqueda de modalidades y recursos necesarios para que la institución pueda elaborar sus propios procesos de cambio, ponerlos en marcha y evaluarlos. (Sánchez Moreno, 1997: 332). El 
Revista de la Escuela de Ciencias de la Educación, año 10, número 9, enero a diciembre de 2014. Páginas 227-246. ISSN 1851-6297. ISSN EN LINEA 2362-3349. EL ASESOR PEDAGÓGICO EN LAS UNIVERSIDADES ARGENTINAS: REALIDADES Y posibilidades de su aCcIONAR. Elisa LuCARELli - Claudia Finkelstein - Viviana Solberg.

rol del asesor pedagógico universitario se configura como mediador de la información y como aquel facilitador en la identificación y resolución de problemas.

La intervención que implican sus acciones hace posible, en un acto reflexivo en común, mirar los problemas desde una nueva mirada.

El análisis de las ponencias incluidas en la investigación, 17 mencionan actividades de asesoramiento; analizadas según destinatarios, se observa un predominio de acciones desarrolladas con la gestión (12 en un total de 17 frecuencias), quedando en un segundo plano las correspondientes a un trabajo directo con las cátedras.

Las acciones de asesoramiento directo en función de las demandas de la gestión se centran prioritariamente en aquellas relativas a los procesos de evaluación y acreditación en las que están comprometidas las instituciones universitarias desde la década del 90 en función de las normativas vigentes en el país. Como expresión de los criterios de política y gestión asociados a conceptos de calidad, estos procesos dan lugar a diversos tipos de participación del asesor pedagógico, desde aquellos más ligados a la incidencia directa de la evaluación y acreditación en la transformación de las prácticas del aula, hasta otros en los que la tarea se evidencia como parte de contribución directa a acciones de la gestión. Así testimonian los asesores pedagógicos:

Seguimiento y Evaluación de la enseñanza [se da] a través de las encuestas a estudiantes y docentes como uno de los aspectos para trabajar la autoevaluación institucional. Con el objetivo de brindar un espacio de apoyo institucional a la docencia, de reflexionar sobre las prácticas de la enseñanza y de contar con un instrumento que permita tomar decisiones sobre la marcha de estos procesos, se diseñó un proyecto de seguimiento de la enseñanza integrando diversas fuentes -la opinión de los estudiantes, de los docentes y planificaciones de las materias; se pretende que el profesor tenga una retroalimentación de su evaluación de modo de permitirle replantear su actividad como docente (UBA- Ing. APU).

En cuanto al sentido que adquiere la práctica del asesor a raíz de su participación en los procesos de evaluación y acreditación, algunos alertan sobre el riesgo de un posible vaciamiento de contenido pedagógico crítico de la tarea.

Otro grupo de equipos (siete sobre doce) testimonian diversos tipos de acciones de asesoramiento directo en función de las demandas de la gestión, más allá de los procesos de evaluación y acreditación y más ligada a procesos pedagógicos y didácticos sustantivos, tales como: los temas curriculares, las estrategias generales de enseñanza, evaluación y promoción, la deserción, el ingreso, siendo los destinatarios principales las Secretarias Académicas, las Comisiones, los Departamentos, los Centros e Institutos de investigación.

En cuanto al asesoramiento a cátedras, principalmente en aspectos pedagógicos y didácticos centrados en los procesos del aula, se encontraron 
Revista de la Escuela de Ciencias de la Educación, año 10, número 9, enero a diciembre de 2014. Páginas 227-246. ISSN 1851-6297. ISSN EN LINEA 2362-3349. EL ASESOR PEDAGÓGICO EN LAS UNIVERSIDADES ARGENTINAS: REALIDADES Y posibilidades de su acCiOnar. Elisa LuCARELli - Claudia Finkelstein - Viviana Solberg.

cinco referencias (sobre ese total de diecisiete). Pareciera que el trabajo directo con los docentes ha ido cobrando menor significación en las tareas de asesoramiento que realizan los equipos pedagógicos, mediándose esa labor a través de las actividades con otros actores (los involucrados en la gestión) y también desplazándose a otras funciones como las de formación docente. Una de ellas sintetiza el propósito de su tarea en esa dimensión de este modo: "Brindar asistencia sistemática e integral en lo que atañe a aspectos de carácter técnico - pedagógico en el nivel Universitario. ... a Cátedras Docentes, Centros e Institutos de la Facultad" (UNSJ- Ing.APU).

\section{Acerca de la investigación}

Partimos del reconocimiento de entender a la investigación científica como una práctica social anclada en un determinado contexto sociohistórico, que tiene su génesis en la problematización de la realidad, y cuya finalidad es generar conocimiento científico con el fin de transformar la realidad (Sirvent, 2006: 6-7). En este sentido el fortalecimiento de la investigación científica se puede constituir en un factor significativo para el mejoramiento de la enseñanza y la formación de profesionales; esto es factible en la medida que se promueva no sólo la participación de estudiantes y docentes en el proceso de creación e invención de nuevos conocimientos, sino que se creen condiciones pedagógicas para la formación en actitudes y habilidades favorables a una concepción constructiva del conocimiento.

En esta línea de pensamiento es posible identificar, según lo expresado en las Comunicaciones analizadas, los proyectos de investigación que desarrollan, en el campo de la Pedagogía y la Didáctica Universitarias, algunos equipos de asesoría pedagógica (trece en total).

Este tipo de investigación manifiesta, en su dinámica y estructura, rasgos propios del campo científico; en esta línea se reconoce que los miembros de los equipos de investigación, que participan del campo, procuran ampliar su capital científico, y toman decisiones observando que los cambios en sus lógicas de trabajo emanan de sus propias necesidades internas. Los asesores pedagógicos así lo reconocen al enfatizar la necesidad de Generar un ámbito de indagación crítica de las problemáticas pedagógicas propias de la institución (UNER; FIng.APU).

Las Comunicaciones presentadas, en lo referente a la dimensión de investigación, expresan consideraciones al lugar y reconocimiento institucional que connota esta práctica, a su grado de institucionalización, a la naturaleza interdisciplinaria de su producción, a los aspectos estratégicos, a sus objetos de estudio, y a aportes que hacen a la conformación del campo de la Pedagogía y la Didáctica Universitaria.

En relación a la respuesta a la demanda institucional hay equipos que manifiestan que la investigación se constituye en un elemento inicial y central 
Revista de la Escuela de Ciencias de la Educación, año 10, número 9, enero a diciembre de 2014. Páginas 227-246. ISSN 1851-6297. ISSN EN LINEA 2362-3349. EL ASESOR PEDAGÓGICO EN LAS UNIVERSIDADES ARGENTINAS: REALIDADES Y posibilidades de su aCcIONAR. Elisa LuCARELli - Claudia Finkelstein - Viviana Solberg.

en las acciones orientadas a enfrentar problemas prioritarios demandados por la institución, constituyéndose la actividad en el eje central y un modus operandi de las acciones que encara la asesoría.

Respecto a la modalidad estratégica se define, en los casos analizados a través, de las formas de articulación en la conformación de los equipos y de la metodología por ellos adoptada. En cuanto al primer aspecto se observa que los equipos se orientan a ser interdisciplinarios e interprofesionales, así como tendientes a incorporar actores extrauniversitarios. Esto permitiría ubicar las acciones que desarrollan los asesores dentro de una perspectiva alejada de la línea tecnicista: en ella este actor define su vínculo con los otros (docentes, comisiones, etc.) desde el lugar del experto que utiliza el conocimiento pedagógico como instrumento de dominación. En los casos analizados el asesor desarrolla una relación de par en un equipo caracterizado por la conjunción de disciplinas singulares, cuyos integrantes son poseedores de distintos saberes que se complementan en el análisis de una práctica de interés común, la formación del estudiante universitario. La multidimensionalidad, nota distintiva de una perspectiva crítica acerca de lo pedagógico (Candau, 1995), se hace presente en esta forma de encarar la asesoría para la realización de proyectos de investigación.

En lo que refiere a las dimensiones metodológicas de los proyectos en sí, que se ponen en juego a la hora de desarrollar el proceso de investigación educativa (Sirvent, 2006) se reconoce en estos casos analizados el predominio de la lógica cualitativa sobre la cuantitativa, cuya meta principal es la comprensión de los hechos que aborda el asesor pedagógico en su quehacer profesional e institucional. Acerca del objeto de estudio y el campo de la Didáctica y la Pedagogía Universitarias nos preguntamos qué investigan los asesores cuando investigan, observando que se define en su dinámica en relación a las demandas institucionales, conformando un abanico que incluye asuntos específicos como la deserción y las tutorías como recurso institucional para prevenirla, la formación de los docentes, la relación entre saber pedagógico y saber disciplinar en la enseñanza, los medios audiovisuales, y otros de carácter más amplio como el modelo pedagógico, las estructuras académicas, la relación educativa, entre otros.

Como conclusión se puede reconocer que la asesoría pedagógica se define a través de su accionar en la institución en función del carácter complejo de su práctica, pues desde este cruce de prácticas que surge como actor central en la construcción de la Didáctica y la Pedagogía universitarias (Lucarelli, 2008). Así parecen reconocerlo los equipos analizados, quienes expresan: "... el asesor "desde" y "en" su práctica de intervención es un constructor de teoría 
Revista de la Escuela de Ciencias de la Educación, año 10, número 9, enero a diciembre de 2014. Páginas 227-246. ISSN 1851-6297. ISSN EN LINEA 2362-3349. EL ASESOR PEDAGÓGICO EN LAS UNIVERSIDADES ARGENTINAS: REALIDADES Y posibilidades de su acCiOnar. Elisa LUCARELLI - Claudia Finkelstein - Viviana Solberg.

en temáticas relacionadas con la formación docente, la didáctica universitaria, así como sobre las problemáticas educativas universitarias" (UNS. SGA.APU).

\section{Acerca de las tareas de extensión}

Hay que señalar que sólo se mencionan tres actividades que corresponden a esta dimensión, referida a las acciones de transferencia directa de la institución universitaria a la comunidad. Ellas dan cuenta de acciones con el nivel medio y terciario, relativas a la capacitación de sus docentes; de articulación con escuelas medias con propósito de orientación de sus estudiantes para la elección de carreras universitarias y de generar proyectos tecnológicos conjuntos para mejorar la situación educativa de jóvenes en situación de riesgo.

\section{Para seguir reflexionando}

Lo presentado hasta el momento da cuenta de la complejidad y diversidad de tareas que desarrolla un asesor pedagógico universitario. El rol del asesor no posee un campo de acción estructurado, sino que el mismo se define en una dinámica entre las condiciones institucionales, la trayectoria de la asesoría en la institución, las posibilidades reales de intervención pedagógica, el trayecto de formación del asesor y su propia representación del rol. El interjuego de todos estos aspectos define el lugar como asesores en una institución, en una permanente dinámica de centración y descentración. Así mismo, la asesoría pedagógica se delinea como un espacio de confluencia y mediación que facilite a través de la cooperación, la integración de un equipo de trabajo que favorezca el estrechamiento de la relación del asesor y los miembros de la comunidad académica en que se inserta.

El encuentro entre pedagogo y profesores docentes expertos en una disciplina supone el encuentro entre dos profesiones, que se ubican en una relación asimétrica, los docentes - profesionales se constituyen en un "nosotros" identificatorio y el pedagogo es un "extranjero" por su historia de formación desposeído de la racionalidad que la especialidad (cualquiera ella sea) brinda. Ambos profesionales son portadores de un conocimiento específico disciplinar, provienen de campos profesionales diferentes, con distintos grados de estructuración, movilidad y con diferentes formas de producción y circulación de los bienes simbólicos.

Desde esta perspectiva, cada institución universitaria presenta características que le son constitutivas, y por ende determina sus peculiares objetivos, modelos de intervención y tareas a las unidades de pedagogía universitaria. 
Revista de la Escuela de Ciencias de la Educación, año 10, número 9, enero a diciembre de 2014. Páginas 227-246. ISSN 1851-6297. ISSN EN LINEA 2362-3349. EL ASESOR PEDAGÓGICO EN LAS UNIVERSIDADES ARGENTINAS: REALIDADES Y posibilidades de su accionar. Elisa LuCARelli - Claudia Finkelstein - Viviana Solberg.

\section{Notas Bibliográficas}

(1) La Lic. Mercedes Lavalleto forma parte del equipo UBA y ha colaborado en la elaboración de este artículo

(2) En su etapa inicial el equipo contó con la colaboración de las siguientes adscriptas a la cátedra de Didáctica de Nivel Superior de la carrera de Ciencias de la educación FFyLUBA: las Lics. Karen Esteve, Vanesa Montenegro, Gisela Piccirillo, Lorena Piliponsky y Noemí Vallejos.

(3) El II Encuentro de Prácticas de Asesoría Pedagógicas Universitarias se realizó en Marzo del año 2010 en la Facultad de Filosofía y letras y en la Facultad de Odontología de la UBA: Fue organizado conjuntamente por el Programa "Estudios sobre el aula universitaria" del Instituto de Ciencias de la Educación de la Facultad de Filosofía y Letras de la UBA y el Área de Educación Odontológica y Asistencia Pedagógica de la Facultad de Odontología de la UBA.

\section{Referencias Bibliográficas}

- $\quad$ Ander Egg, E. (1986). Hacia una pedagogía autogestionaria. Bs. As: Ed. Humanitas.

- Cáceres, M. y otros (2003). La formación pedagógica de los profesores universitarios. Revista Iberoamericana de Educación Universidad de Cienfuegos. Recuperado de http:// www.rieoei.org/deloslectores/475Caceres.pdf

- $\quad$ Candau, V. M.(org.) (1995). Rumo a uma nova didática. Petrópolis: Vozes.

- Coria A. y Edelstein, G. (1993, nov.). El pedagogo en la universidad. Un discurso posible. Revista Pensamiento Universitario. Año 1. No 1.

- Da Cunha M. I. y Leite, D. (2006). Decisões pedagógicas e estructuras de poder na universidade. Campinas: Papirus Editora.

- Da Cunha M. I. (comp.) (2010). Trajetorias e lugares de Formaçao da docencia universitaria: da perspectiva individual ao espacio institucional. Araraquara: Junqueira \& Martin ed.

- Da Cunha M. I. y Lucarelli, E. (2011). Fundamentación del Proyecto Conjunto de Investigación. Mercosur Argentina (SPU)- Brasil (CAPES) PPCP 003/11. Estrategias institucionales para el mejoramiento de la calidad de la Educación Superior y el desarrollo profesional docente. Buenos Aires: FFyL.UBA.

- Fernández, L. (2000). Prólogo. En: Lucarelli, E. (Comp.). El asesor pedagógico en la universidad: de la teoría pedagógica a la práctica en la formación. Buenos Aires: Paidós.

- Fernández Lamarra, N. (2003). La educación superior argentina en debate. Situación, problemas y perspectivas. Buenos Aires: EUDEBA.

- Finkelstein, C. y Faranda, C. (2000): Hacia la construcción del Asesor Pedagógico en la Universidad. En Lucarelli, E. (comp.). El asesor pedagógico en la Universidad. De la teoría a la práctica en la formación. Buenos Aires: Paidós.

- $\quad$ Finkelstein, C., Gardey, M., Amantea, A.; Rodríguez, G. Y Rozenblum, G. (2011). La preeminencia de la articulación teoría-practica en los dispositivos de formación de la Carrera Docente de la Facultad de Odontología de la UBA. Ponencia presentada en VI Jornadas Nacionales sobre la Formación del Profesorado. Mar del Plata. Argentina

- $\quad$ Finkelstein, C. (2012). ¿Los Docentes Universitarios, pueden Innovar en sus Prácticas? En G. Volpatoy M. Moog Pinto (Org.) Pedagogia Universitária: olhares e percepções. Curitiba: PR, Editora CVR.

- $\quad$ Frondizi, R. (2003). La universidad en un mundo de tensiones. Misión de las universidades en América Latina. Buenos Aires: EUDEBA. 
Revista de la Escuela de Ciencias de la Educación, año 10, número 9, enero a diciembre de 2014. Páginas 227-246. ISSN 1851-6297. ISSN EN LINEA 2362-3349. EL ASESOR PEDAGÓGICO EN LAS UNIVERSIDADES ARGENTINAS: REALIDADES Y POSibilidAdes de su ACCIONAR. Elisa LUCARELLI - Claudia FinKelstein - Viviana Solberg.

- Gardey, M., Finkelstein, C., Amantea, A. y Rosenblum, G. (2008, junio). La asesoría pedagógica en la Universidad de Buenos Aires. Análisis de un caso particular: Facultad de Odontología. Ponencia presentada en el Primer Encuentro Nacional de prácticas de asesorías pedagógicas universitarias. Facultad de Ciencias Bioquímicas y Farmacéuticas. Universidad Nacional de Rosario.

- Gómez López, L. F. (2008, Julio - setiembre). Los determinantes de la práctica educativa. Revista Universidades UDAL $N^{\circ}$ 38. (pp. 29-39). Recuperado de http://www.redalyc. org/pdf/373/37303804.pdf

- Ickowicz, M. (2004). La formación de profesores en la Universidad: avances de investigación sobre los trayectos de formación para la enseñanza en profesores universitarios sin formación docente de grado. Revista del IICE. Año XII. № 22.

- Langer, A. (2008). El sistema científico y las universidades: revisión de teorías y enfoques en América Latina y Argentina. En G. Riquelme (ed.) Las universidades frente a las demandas sociales y productivas. Buenos Aires: Miño y Dávila. Tomo I.

- Larramendy, A. (2012). La asesoría pedagógica universitaria y la orientación al estudiante. En E. Lucarelli y C. Finkelstein. El asesor pedagógico en la Universidad: desafíos, perspectivas, producciones. Buenos Aires: Miño y Dávila.

- Lucarelli, E. (Comp.) (2000). El asesor pedagógico en la universidad: de la teoría pedagógica a la práctica en la formación. Argentina: Paidós.

- Lucarelli, E. y Finkelstein, C. (2012). El asesor pedagógico en la Universidad: desafíos, perspectivas, producciones. Argentina: Miño y Dávila.

- Lucarelli, E. (2008, diciembre). Problemáticas en torno a un rol: El asesor pedagógico universitario. Ponencia presentada en $\mathrm{X}$ Congreso Interuniversitario de Organización de Instituciones Educativas. Barcelona. España

- Murillo Estepa, P. (1997). El diseño de la función asesora en el nuevo sistema educativo: posibilidades y problemas. En: J. López Yáñez y C. Marcelo García (coords): Asesoramiento curricular y organizativo en educación. Barcelona: Ariel.

- Murillo Estepa, P. (2008, mayo). ¿Ayuda u obstáculo? Sentido y credibilidad de la función asesora. Revista Profesorado. 121- art.9. Recuperado de http://www.ugr.es/ recfpro/ rev121ART9.pdf

- $\quad$ Sánchez Moreno, M. (1997). El proceso de asesoramiento. En: M. García y J. López Yañez (Coords.) Asesoramiento curricular y organizativo en educación. Barcelona: Ariel.

- Sánchez Núñez (2001). El desarrollo profesional del docente universitario Revista Universidades UDAL $N^{\circ}$ 22. Recuperado de http://www.udual.org/CIDU/ Revista/22/DesarrolloProfesional.htm

- $\quad$ Sirvent, M. T. (1985). Nuestra propuesta de trabajo: producto de una tarea compartida. Boletín del ICE. Buenos Aires No 3.

- Villalobos Clavería, A. y Melo Hermosilla, Y. (2008). La formación del profesor universitario: Aportes para su discusión. Revista Universidades UDAL N 39. pp. 3-20. Recuperado de http://www.udual.org/CIDU/Revista/39/revista39.pdf 